\title{
Comparison of Diabetic Neuropathy Symptom Score and Medial Plantar Sensory Nerve Conduction Studies in Diabetic Patients Showing Normal Routine Nerve Conduction Studies
}

\author{
Jae Young An ${ }^{1}$, Min Su Park ${ }^{2}$, Joong Seok Kim ${ }^{1}$, Young Min Shon ${ }^{1}$, Seung Jae Lee ${ }^{1}$, \\ Yeong In Kim ${ }^{1}$, Kwang Soo Lee ${ }^{1}$ and Byoung Joon Kim ${ }^{3}$
}

\begin{abstract}
Objective Diabetic Neuropathy Symptom (DNS) scoring and medial plantar NCS are useful methods for diagnosis of diabetic polyneuropathy (DPN). We evaluated the correlation between DNS score and medial plantar NCS in diabetic patients with a normal routine NCS.

Method Nineteen healthy subjects were included as a control group. Fifty patients with diabetes mellitus who were referred for the evaluation of DPN were recruited (35 asymptomatic and 15 symptomatic). Control subjects and diabetic patients over the age of 70 were excluded from this study.

Results Medial plantar nerve action potential (NAP) was recordable in all 19 control subjects and was not obtainable in 7 out of 15 symptomatic patients and in 5 out of 35 asymptomatic patients. The amplitudes of sural (Spearman $r=-0.293, p=0.003$ ) and medial plantar NAP (Spearman $r=-0.215, p=0.03$ ) correlated with DNS score.

Conclusion An abnormality finding in the medial plantar sensory nerve conduction study is a more sensitive indicator than sural nerve conduction study in the diagnosis of DPN and the medial plantar sensory nerve should be included in the evaluation of DPN in patients showing normal routine NCS.
\end{abstract}

Key words: Diabetic polyneuropathy, Diabetic Neuropathy Symptom (DNS) score, Medial plantar sensory nerve

(Inter Med 47: 1395-1398, 2008)

(DOI: 10.2169/internalmedicine.47.0901)

\section{Introduction}

Early detection of diabetic polyneuropathy (DPN) is important due to the possibility of early intervention. The Diabetic Neuropathy Symptom (DNS) scoring system is a simple and easy system that can be used to discriminate between patients with and without DPN $(1,2)$. Although nerve conduction studies (NCS) are commonly used tests, the results of routine NCS are normal in 38 to $67 \%$ of patients with suspected DPN $(3,4)$. However, the medial plantar sensory nerve, which is a more distal nerve than the sural nerve, is known to be more sensitive to the detection of sen- sory neuropathy $(3,5-8)$.

This study was carried out in order evaluate the usefulness of medial plantar sensory NCS and DNS scores and to investigate the correlation between both diagnostic categories in diabetic patients with normal routine NCS.

\section{Methods}

\section{Patients and controls}

Nineteen healthy individuals (9 males and 10 females; age 30-64 years) were included in the present study. The exclusion criteria were subjects over 70 years of age, history of

${ }^{1}$ Department of Neurology, The Catholic University of Korea, Seoul, Korea, ${ }^{2}$ Department of Neurology, National Cancer Center of Korea, Goyang, Korea and ${ }^{3}$ Department of Neurology, Sungkyunkwan University School of Medicine, Samsung Medical Center, Seoul, Korea Received for publication January 9, 2008; Accepted for publication May 2, 2008 Correspondence to Dr. Byoung Joon Kim, bjkim@skku.edu 
Table 1. Clinical and Laboratory Findings of Diabetic Patients and Controls

\begin{tabular}{|c|c|c|c|c|c|c|}
\hline & \multicolumn{2}{|c|}{ Diabetic patients } & \multicolumn{4}{|c|}{$\mathrm{p}$ value } \\
\hline & Symptomatic (1) & Asymptomatic (2) & Control(3) & 1 vs. 2 & 1 vs. 3 & 2 vs. 3 \\
\hline Number & 15 & 35 & 19 & & & \\
\hline Mean age (years) & $54.3 \pm 12.9$ & $51.0 \pm 8.7$ & $50.0 \pm 9.01$ & 0.045 & 0.019 & ns \\
\hline DM duration (months) & $138.8 \pm 90.7$ & $74.5 \pm 68.2$ & & 0.000 & & \\
\hline $\operatorname{Sex}(\mathrm{F} / \mathrm{M})$ & $10 / 5$ & $20 / 15$ & $9 / 10$ & 0.049 & ns & ns \\
\hline Fasting glucose (mg/dL) & $154.4 \pm 41.3$ & $156.5 \pm 56.0$ & & ns & & \\
\hline $2-$ hour glucose $(\mathrm{mg} / \mathrm{dL})$ & $226.5 \pm 72.8(n=13)$ & $236.2 \pm 66.4(n=27)$ & & ns & & \\
\hline HbA1c $(\%)$ & $8.1 \pm 2.1$ & $8.5 \pm 2.1(n=34)$ & & ns & & \\
\hline DNS score & $1(n=11), 2(n=4)$ & $0(n=35)$ & & & & \\
\hline Autonomic symptoms ${ }^{\dagger}$ & $5 / 15$ & $0 / 35$ & & 0.000 & & \\
\hline
\end{tabular}

Data are means \pm SD. DNS score, Diabetic Neurpathy Symptom score; ns, not significant

${ }^{\dagger}$ Autonomic symptoms include orthostatic hypotension, impotence, urinary symptoms and night diarrhea.

the intake of potentially neurotoxic drugs, lumbosacral radiculopathy or general medical problems with the potential involvement of the peripheral nervous system.

Eighty-two diabetic patients who underwent electromyography for the evaluation of DPN were recruited. The exclusion criteria included: 1) asymmetric complaints of paresthesia in the distal lower extremities, 2) asymmetric sensory impairment or asymmetric decreased/absent tendon reflexes, 3) patients over the age of 70 years old, 4) patients with any other cause of neuropathy except for diabetes. Among symptomatic diabetic patients, 11 of 15 patients with a DNS score of $1(73.3 \%)$, and 4 of 12 patients with a score of $2(33.3 \%)$ had normal routine NCS results. Fifty diabetic patients with normal routine NCS (25 males and 25 females, age 26-69 years, 35 asymptomatic and 15 symptomatic, 2 type 1 diabetes, 48 type 2 diabetes) were included. All subjects gave their written informed consent to participation in this study.

\section{DNS score}

DNS score is a four-item validated symptom scoring system based on unsteadiness in walking, neuropathic pain, paresthesia, and numbness. The presence of one symptom was scored as 1 point. A score of 1 or higher was defined as positive for DPN.

\section{Nerve conduction studies}

NCS was performed with standard surface stimulation and recording techniques using an electromyography type $\mathrm{Ni}$ colet Viking IV with standard filter settings. Routine NCS included motor and sensory nerve conduction in one median and one ulnar nerve, sensory nerve conduction in the bilateral sural nerves, motor nerve conduction in one peroneal and one posterior tibial nerve and F-waves in the median, ulnar, peroneal and posterior tibial nerves. Abnormalities were judged according to the age-matched normal values (9). We also performed medial plantar sensory NCS using surface recording as previously described (10). Briefly, an active surface electrode is placed above the flexor retinacu- lum and medial to the medial malleolus and 100 responses are averaged with a signal averager. A ring electrode is used on the great toe to stimulate the medial plantar nerve. The surface skin temperature on the foot was controlled at $32^{\circ} \mathrm{C}$. Medial plantar sensory NCS was considered abnormal in patients who scored 2 SD below or above the mean of the control group (Table 2).

\section{Statistical analysis}

Clinical and electrophysiological measures were compared using chi-square tests for proportions and Mann-Whitney Utests for the comparison of means. $\mathrm{p}<0.05$ was considered significant.

\section{Results}

The symptomatic patients were older, had a longer duration of diabetes and more autonomic symptoms than the asymptomatic patients. Fasting, 2-hour glucose and the HbA1c levels did not differ between symptomatic and asymptomatic patients (Table 1).

Medial plantar sensory nerve action potential (NAP) was recordable in all 19 control subjects. The amplitude of medial plantar sensory NAP (Spearman $\mathrm{r}=-0.425, \mathrm{p}=0.008$ ) and sural NAP (Spearman $r=-0.326, p=0.045$ ) in the control subjects decreased with age. Five patients with a DNS score of $0(14.3 \%), 5$ with a score of $1(45.4 \%)$, and 2 with a score of $2(50 \%)$ had abnormal medial plantar sensory NAP. The amplitude of the sural NAP (Spearman $r=-0.293, p=0.003$ ) and medial plantar NAP (Spearman $\mathrm{r}=-0.215, \mathrm{p}=0.03$ ) correlated with the DNS score (Fig. 1). The electrophysiological findings in the control and diabetic patients are described in Table 2.

\section{Discussion}

When diagnosing neuropathy, the San Antonio Consensus recommends at least one measurement in five different diagnostic categories (11). But this systematic evaluation is ex- 
Table 2. Electrophysiological Findings of Diabetic Patients and Control Group

\begin{tabular}{|c|c|c|c|c|c|c|}
\hline & \multicolumn{2}{|c|}{ Diabetic patients } & \multirow[b]{2}{*}{ Control(3) } & \multirow[b]{2}{*}{1 vs. 2} & \multicolumn{2}{|l|}{$\mathrm{p}$ value } \\
\hline & Symptomatic (1) & Asymptomatic(2) & & & 1 vs. 3 & 2 vs. 3 \\
\hline Number & 15 & 35 & 19 & & & \\
\hline Plantar NAP amplitude $(\mu \mathrm{V})$ & $0.79 \pm 0.84$ & $1.23 \pm 0.9$ & $1.71 \pm 0.84$ & 0.044 & 0.000 & 0.007 \\
\hline plantar CV (m/s) & $20.8 \pm 20.0$ & $33.6 \pm 14.4$ & $39.8 \pm 3.8$ & 0.008 & 0.000 & 0.033 \\
\hline $\begin{array}{l}\text { Abnormal plantar NAP: } N \\
(\%)\end{array}$ & $7 / 15(46.7 \%)$ & $5 / 35(14.3 \%)$ & & 0.002 & 0.000 & 0.014 \\
\hline Sural NAP amplitude $(\mu \mathrm{V})$ & $12.0 \pm 5.2$ & $17.4 \pm 8.5$ & $21.4 \pm 7.0$ & 0.000 & 0.000 & 0.002 \\
\hline Sural CV $(\mathrm{m} / \mathrm{s})$ & $39.9 \pm 3.4$ & $40.7 \pm 3.0$ & $42.7 \pm 1.8$ & ns & 0.000 & 0.000 \\
\hline
\end{tabular}

Data are means \pm SD. CV, conduction velocity; NAP, nerve action potential; ns, not significant

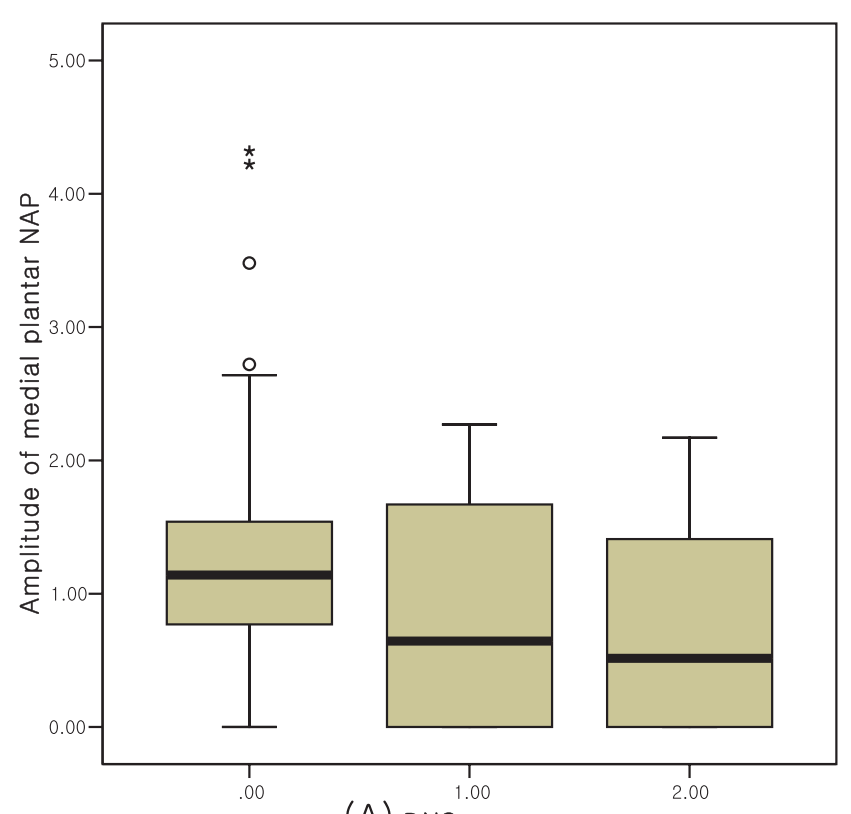

(A) DNSscore

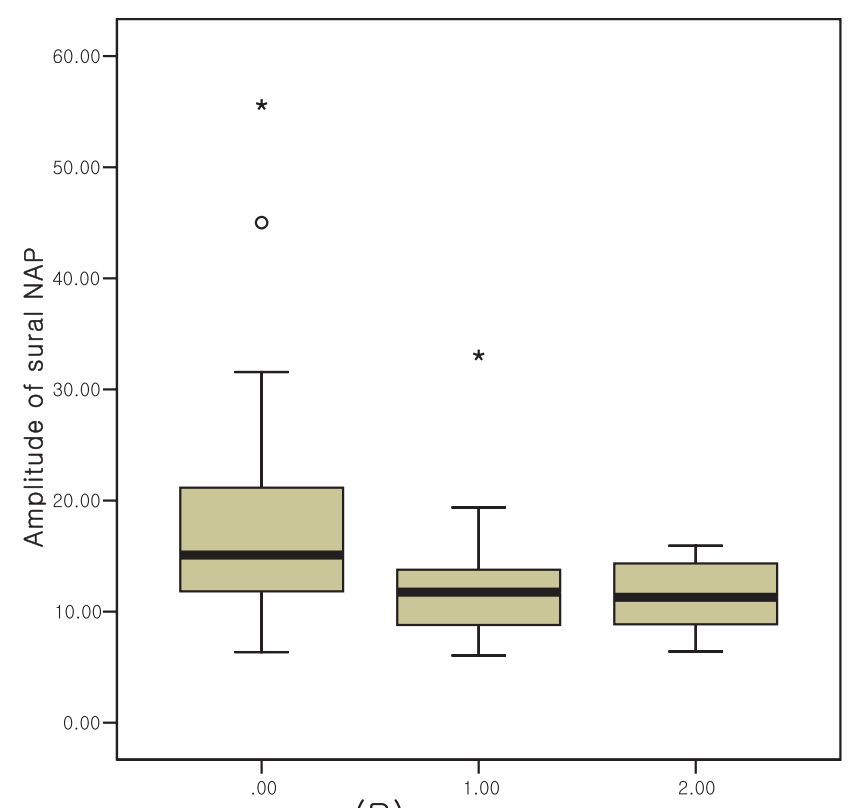

(B) DNSscore

Figure 1. Correlation between DNS score and amplitude of medial plantar sensory (Spearman $\mathbf{r}=-\mathbf{0 . 2 1 5}, \mathrm{p}=\mathbf{0 . 0 3})(\mathrm{A})$ and sural NAP (Spearman $r=-0.293, p=0.003$ ) (B) in diabetic patients. DNS score: Diabetic Neuropathy Symptom score, NAP: nerve action potential. pensive and time-consuming. EMG and NCS are often the most useful initial laboratory studies in the evaluation of a patient with peripheral neuropathy $(11,12)$.

However, the most commonly involved segments, such as the feet, are not tested in routine NCS. Routine NCS is of limited value for the evaluation of DPN, especially in the early stage. Medial plantar sensory NCS at the surface or near the needle recording site can provide a more sensitive diagnosis of DPN. Although there were concerns about the effects of age, several studies showed that medial plantar sensory NAP was elicited in all healthy control subjects under the age of $70(12,13)$. Therefore, patients and control subjects over the age of 70 were excluded from this study.

Although there are significant differences in the sural NCS value between control subjects and asymptomatic or symptomatic patients, the sural nerve of diabetic patients is still in the normal range. Medial plantar sensory NAP was abnormal in $46.7 \%$ of symptomatic and $14.3 \%$ of asymptomatic diabetic patients with normal routine NCS in this study. These results indicate that axonal degeneration in the medial plantar nerve, which is a predominant pathological finding in DPN (14-17), may precede that of the sural nerve.

The drawback of the interpretation of these electrophysiological results is that symptomatic patients were significantly older than asymptomatic patients. Age is an important factor affecting results of medial plantar sensory NCS and further research with a larger sample size is needed to control the influence of age on NCS. But previous studies revealed that medial plantar sensory NAP was elicited in all healthy control subjects under the age of $70(12,13)$. And the effect of age on our NCS results may be minimal because medial plantar sensory NAP was not provoked in all diabetic patients with abnormal medial plantar sensory NCS.

Although symptomatic patients had more autonomic symptoms than asymptomatic patients, we do not know whether or not small fiber neuropathy has relevance to medial plantar sensory NCS because a diagnostic work-up of small fiber neuropathy was not performed, that is one of limitation of our study.

The medial plantar sensory NCS may be helpful in the diagnosis of subclinical DPN in asymptomatic diabetic patients. And so, medial plantar sensory NCS should be per- 
formed irrespective of the patient's symptoms when evaluating diabetic patients, especially with normal routine NCS.

DNS score is fast and easy to perform in outpatient clinics (1). Abnormal medial plantar NAP in symptomatic patients was considerably more frequent than in asymptomatic patients. The amplitude of medial plantar sensory NAP correlated with DNS score. Thus, the four simple questions used in DNS scoring may allow for the easy diagnosis of DPN in outpatient clinics. The small number of patients with DNS scores of 2 or more showing normal routine NCS is a limitation of our study, and it means that a DNS score of 2 or more was associated with an increased prevalence of abnormal routine NCS results. Based on the results of this study, we recommend medial plantar NCS for the evaluation for patients with a DNS score of 1 .

Easy screening and early identification of the DPN by DNS scoring and medial plantar sensory NCS offer an opportunity for the patient with diabetes to actively correct their poor glycemic control and it can be used to identify higher risk patients that should receive closer attention before the onset of significant morbidity.

\section{References}

1. Meijer JW, Bosma E, Lefrandt JD, et al. Clinical diagnosis of diabetic polyneuropathy with the Diabetic Neuropathy Symptom and Diabetic Neuropathy Examination scores. Diabetes Care 26: 697701, 2003.

2. Meijer JW, Smit AJ, Sonderen EV, Groothoff JW, Eisma WH, Links TP. Symptom scoring systems to diagnose distal polyneuropathy in diabetes: the Diabetic Neuropathy Symptom score. Diabet Med 19: 962-965, 2002.

3. Nodera H, Logigian EL, Herrmann DN. Class of nerve fiber involvement in sensory neuropathies: clinical characterization and utility of the plantar nerve action potential. Muscle Nerve 26: 212217, 2002.

4. Periquet MI, Novak V, Collins MP, et al. Painful sensory neuropathy: prospective evaluation using skin biopsy. Neurology 53: 1641-1647, 1999.

5. Ponsford SN. Sensory conduction in medial and lateral plantar nerves. J Neurol Neurosurg Psychiatry 51: 188-191, 1988.

6. Reeves ML, Seigler DE, Ayyar DR, Skyler JS. Medial plantar sensory response. Sensitive indicator of peripheral nerve dysfunction in patients with diabetes mellitus. Am J Med 76: 842-846, 1984.

7. Herrmann DN, Ferguson ML, Pannoni V, Barbano RL, Stanton M, Logigian EL. Plantar nerve AP and skin biopsy in sensory neuropathies with normal routine conduction studies. Neurology 63: 879-885, 2004.

8. Oh SJ, Melo AC, Lee DK, et al. Large-fiber neuropathy in distal sensory neuropathy with normal routine nerve conduction. Neurology 56: 1570-1572, 2001.
9. Oh SJ. Clinical Electromyography: Nerve Conduction Studies. 3nd ed. Williams and Wilkins, Baltimore, MD, 2003: 86-106.

10. Oh SJ, Sarala PK, Kuba T, Elmore RS. Tarsal tunnel syndrome: electrophysiological study. Ann Neurol 5: 327-330, 1979.

11. American Diabetes Association, American Academy of Neurology. Consensus Statement: Report and recommendations of the San Antonio Conference and Diabetic Neuropathy. Diabetes Care 11: 592-597, 1988.

12. Lee KW, Oh SJ. Early appearance of aging phenomenon in the interdigital nerves of the foot. Muscle Nerve 17: 58-63, 1994.

13. Løseth S, Nebuchennykh M, Stålberg E, Mellgren SI. Medial plantar nerve conduction studies in healthy controls and diabetics. Clin Neurophysiol 118: 1155-1161, 2007.

14. Brown MJ, Martin JR, Asbury AK. Painful diabetic neuropathy. Arch Neurol 33: 164-171, 1976.

15. Llewelyn JG, Gilbey SG, Thomas PK, King RH, Muddle JR, Watkins PJ. Sural nerve morphometry in diabetic autonomic and painful sensory neuropathy. A clinicopathological study. Brain 114: 867-892, 1991.

16. Said G, Slama G, Selva J. Progressive centripetal degeneration of axons in small fiber diabetic polyneuropathy. Brain 106: 791-807, 1983.

17. Uncini A, De Angelis MV, Di Muzio A, et al. Chronic inflammatory demyelinating polyneuropathy in diabetics: motor conductions are important in the differential diagnosis with diabetic polyneuropathy. Clin Neurophysiol 110: 705-711, 1999.

(C) 2008 The Japanese Society of Internal Medicine http://www.naika.or.jp/imindex.html 